\title{
Combination Adaptive Traffic Algorithm and Coordinated Sleeping in Wireless Sensor Network
}

\author{
M. Udin Harun Al Rasyid, Eka Saputra Aji, Isbat Uzzin Nadhori \\ Politeknik Elektronika Negeri Surabaya \\ Electronic Engineering Polytechnic Institute of Surabaya \\ Email: udinharun@pens.ac.id, ekapens@student.eepis-its.edu, isbat@pens.ac.id
}

\begin{abstract}
Wireless sensor network (WSN) uses a battery as its primary power source, so that WSN will be limited to battery power for long operations. The WSN should be able to save the energy consumption in order to operate in a long time.WSN has the potential to be the future of wireless communications solutions. WSN are small but has a variety of functions that can help human life. WSN has the wide variety of sensors and can communicate quickly making it easier for people to obtain information accurately and quickly. In this study, we combine adaptive traffic algorithms and coordinated sleeping as power-efficient WSN solution. We compared the performance of our proposed ideas combination adaptive traffic and coordinated sleeping algorithm with non-adaptive scheme. From the simulation results, our proposed idea has good-quality data transmission and more efficient in energy consumption, but it has higher delay than that of non-adaptive scheme.
\end{abstract}

Keywords:WSN,adaptive traffic,coordinated sleeping,beacon order,superframe order.

\section{INTRODUCTION}

With recent advances in micro-electronics technology and wireless communication technology, wireless sensor networks (WSN) have attracted attention as one of the communication systems. WSN generally operate with limited battery power due to limited funds and the size of the chassis, therefore WSN asked to consume low energy in achieving a long operation without battery recharges.

In WSN, the node does not transmit data too often to make their radio interfaces in an inactive state as long as possible in order to save the energy consumption.IEEE 802.15.4 MAC Protocol do a power saving mechanism which determines the degree of activity cycle is active or inactive on the sensor node in WSN. Although the cycle should be reduced as much as possible to lower power consumption in each node, this also affects the performance of data transmission, such as throughput and transmission 
delay. Therefore, we need to determine the length of the cycle with consideration of energy consumption and transmission performance [1].

On the other hand, the characteristic of traffic on WSN is far different from other wireless systems such as cellular network and Local Area Network (LAN). In WSN, the traffic load increases temporally considered when all sensor nodes transmit data. As a result, the distribution of traffic over time will vary greatly. Moreover, from the standpoint of traffic spatial or geographical distribution, WSN will have a distribution of non-uniform traffic in the entire coverage area. From the above observations, in the IEEE 802.15.4 MAC cycle should be determined by considering the traffic behavior in the temporal and geographic distribution [1].

Wireless sensor network is used to monitor and control the physical environment from remote location. The sensor nodes operate using limited energy. Traffic patterns on WSN is sporadic and does not follow a specific pattern. In this paper, we examine the energy efficiency of the medium access control protocol (MAC) which is adaptive to the traffic situation on the sensor network application using adaptive traffic algorithms and coordinated sleeping. Traffic information is available on the queue node, then dynamically change the length of the active periodand sleep period in a sensor node. Thispaper will compare the performance of non adaptive WSN to the adaptive traffic WSN.

\section{RELATED WORKS}

Kazuo MORI, et al.[2] proposed traffic adaptation by controlling the superframe order(SO) to modify the superframe structure. In the adaptive traffic in IEEE 802.15.4, each node monitors the transmission queue status to estimate trafficload at any given moment. Monitoring of the queue is done each time when a node will send a packet to another node. After queue monitoring, if the queue slot occupancy rate greater than a predetermined level, the node will report the current status of the queue by sending a control packet called Queue Status Indicator (QSI) to the PAN coordinator to request modification the structure of the PAN superframe. Coordinator receive QSIpackets will immediately maximize the active part. If the PAN coordinator does not receive packets QSI for at least a predetermined superframe after receiving the last QSI packets will reduce the active part.

Marwa et al. [3] proposed an adaptive algorithm to improve network performance. The performance of the beacon mode is essentially influenced by the parameters of the beacon frame, which is the Beacon Order (BO) and superframe order (SO). These two parameters determine the active and inactive phases of the nodes and also determine the duty cycle of the nodes. Values of BO and SO should be determined properly because the MAC standard parameters can degrade the performance of WSN. This study simulates the WSN with a beacon mode to find the values of BO and SO in a star topology with various number of nodes. To determine the quality of the network will be evaluated in several parameters, energy consumption, 
throughput and delay.The new algorithm is able to reduce energy consumption by $7 \%$, reduce delay by $26 \%$ and increase the throughput by $16 \%$.

Wei Yeet al.[4] proposed energy savings and automatic configuration mechanism, this study filed S-MAC, the MAC protocol designed for WSN. WSN uses the battery as energy source. Sensor nodes will experience an inactive state in the long time. Nodes will be active when something is detected quickly. To reduce the energy consumption of the phase listen to stationary phase, nodes with scheduling system periodically will go into the stationary phase. This study evaluates the S-MAC with some sample nodes. The results showed a standard MAC consumes 2-6 times more energy than S-MAC in trafficload sent every 1-10 seconds.

Huang et al. [5] developed an analytical model describing the operation of various versions of IEEE $802.16 \mathrm{e} / \mathrm{m}$ sleep-mode power-saving mechanisms and study their connection dropping probabilities and energy saving efficiencies by using the dynamic base station cluster scheme to improve the performance of the existing sleep-mode schemes. Al-Anbagi et al. [6] proposed an adaptive scheme that can achieve low latency while maintaining high reliability values in cluster-tree based WSNs. The scheme provides Quality of Service (QoS) differentiation to high priority data in order to accommodate high packet arrival rates with low latency. Zhuo et al. [7] proposed iQueue-MAC to provide immediate yet energy-efficient throughput enhancement for dealing with burst or heavy traffic. iQueue-MAC uses of queue length of each sensor node and allocates suitable TDMA slots to them for packets transmission. During light traffic period, no extra slots will be allocated, While in burst or heavy traffic period, iQueue-MAC senses the build up of packet queues and dynamically schedules adequate number of slots for packet transmission.

From the previous works mentioned above, adaptive traffic algorithm for IEEE 802.15.4 standard are proposed in [1-2] and [6-7]. The sleeping adaptive algorithm for IEEE 802.11 and IEEE 802.16 are proposed in [4] and [5], respectively, that the standard is different to IEEE 802.15.4. In this paper, we adopt the idea of adaptive sleeping from [4] and [5], then combined to adaptive traffic algorithm.

\section{ORIGINALITY}

WSN is a new technology for wireless communication. WSN has the potential to be one of future communication systems because ofability to solve a variety of problems, especially in the scope of wireless networks. Sensor nodes in WSN in general consumes low power because of primary power source is battery, thusWSN requested energy consumption as low as possible to operate with a long time.

We propose the combination of adaptive traffic algorithms and coordinatedsleepingas the solution to make energy savings in the WSN. We 
combine the concept of adaptive traffic and coordinated sleeping concept to produce a good network performance with low energy consumption.

\section{SYSTEM DESIGN}

Network topology is the arrangement of the various elements (links, nodes, etc.) of a network. Basically, the network topology can be described physically or logically. Physical topology refers to the placement of the various components of the network, including the device location and cable installation. While the logical topology shows how data flows in the network, the distance between nodes, physical interconnections, transmission rates, and the types of signals that may differ between networks.

In this paper, a logical network topology applied, namely the observation of changes in the network with quality of service parameters (throughput, delay, energy, and the probability of successful packet) in a wireless sensor network. We use a cluster topologyin this study. There are a PAN coordinator, full function device(FFD)node and reduced function device(RFD)node in a cluster topology as shown in Figure 1. The network will have the network in clusters so that some clusters. Each cluster contained 1 Cluster Head $(\mathrm{CH})$ that will be connected with one PAN Coordinator. In each cluster there are some node which connected to $\mathrm{CH}$. Cluster Head is a Full Function Device (FFD) and Reduced Function Device nodes form (RFD). Nodes can be interconnected with other nodes in the cluster or different clusters.

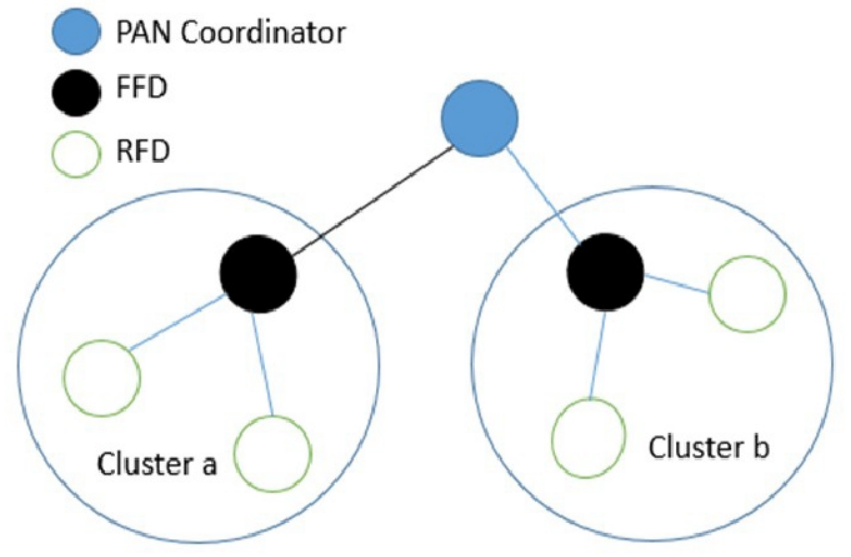

Figure 1. Cluster Topology

The purpose of this paper is to combine the concept of the adaptive traffic and coordinated sleeping concept to be applied to WSN. To know the result of the combination, we perform simulations using NS2. The concept of adaptive traffic enables the superframe order (SO) and beacon order (BO) values change to reflect the network traffic (trafficload). To do this test done SO and BO values ranging from 3 to 10 is applied to the concept of coordinated sleeping SO and BO configuration. Value of SO and BO at PAN, Cluster Head, and different sensor node, this configuration implements the 
concept of coordinated sleeping. SO and BO configuration to implement the concept of coordinated sleeping as shown in Figure 2. Simulation will generate .TR file, the file is then processed by awk file to generate the value of the performance of WSN. Performance results of a simulation throughput, energy consumption and delay. Both the poor quality of the network can be represented by the value of the throughput. The maximum value of each trafficload be taken as adaptive traffic. Combination of steps can be seen in Figure 3.

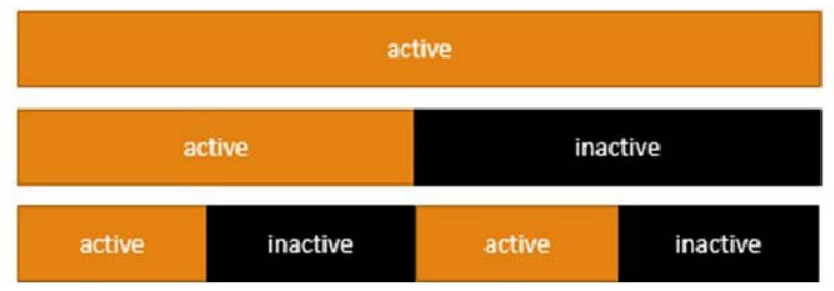

\begin{tabular}{|c|c|c|}
\hline PAN & $=$ & BO PAN=SO PAN \\
\hline $\begin{array}{l}\text { Cluster } \\
\text { Head }\end{array}$ & $=$ & $\begin{array}{l}\mathrm{BO} \mathrm{CH}=\mathrm{BO} \text { PAN, } \\
\text { SO } \mathrm{CH}=\mathrm{BO} \mathrm{CH}-1\end{array}$ \\
\hline $\begin{array}{l}\text { Node } \\
\text { Sensing }\end{array}$ & $=$ & $\begin{array}{l}\text { BO Node }=\mathrm{BO} \text { CH, } \\
\text { sO Node }=\mathrm{BO} \text { Node- } 1\end{array}$ \\
\hline
\end{tabular}

Figure 2. Coordinated Sleeping Concept

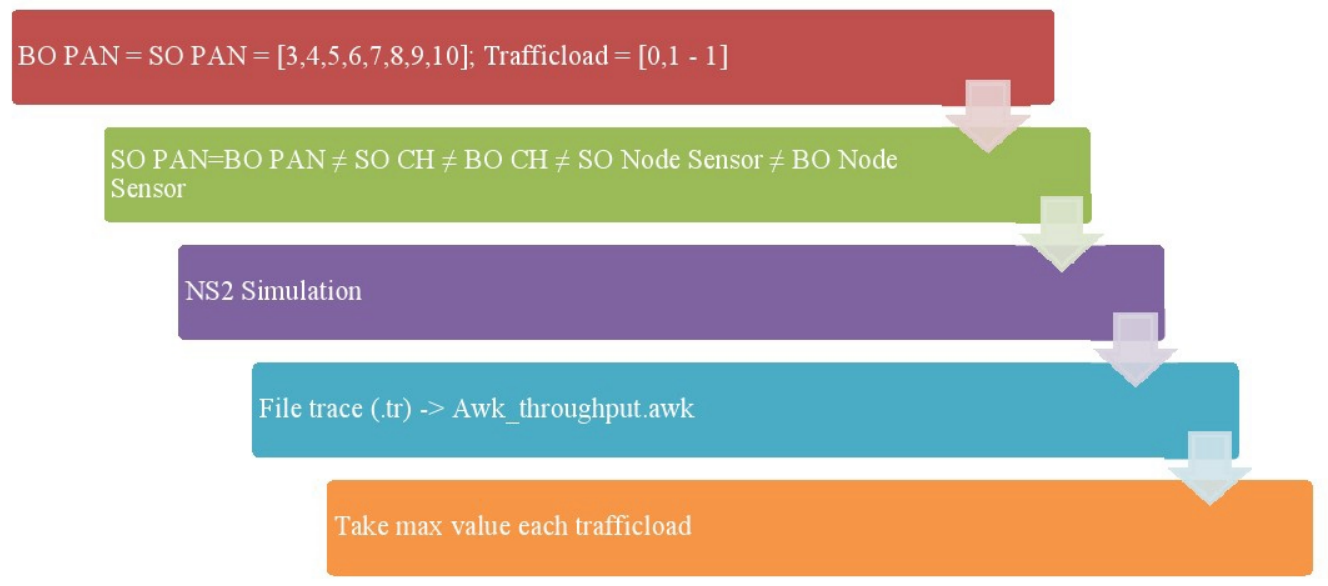

Figure 3. Combination Steps

From figure 2 and figure 3, we decide adaptive traffic and coordinated sleeping as follow, the first initialization for $\mathrm{BO}$ and SO PAN is equal to 8:

1) $\mathrm{BO}$ and $\mathrm{SO} \mathrm{PAN}=8$

2) At trafficload 0,4; BO and SO PAN is decreased by 1

3) At trafficload 0,5; BO and SO PAN is increased by 1

4) At trafficload 0,7; BO and SO PAN is increased by 1

5) At trafficload 0,9; BO and SO PAN is decreased by 2

6) At trafficload 1; BO and SO PAN is increased by 2

7) From step 2-6, the current value of $\mathrm{BO}$ and SO PAN for others trafficload is equal to the previous value of $\mathrm{BO}$ and $\mathrm{SO}$, then the value of BO and SO for cluster head and node sensing follow coordinated sleeping concept as shown in figure 2 . 


\section{EXPERIMENT AND ANALYSIS}

To make the WSN simulation, we need to set the parameters. Table 1 contains the parameters for the simulation parameters of this paper.

Table 1. Simulation Parameters

\begin{tabular}{|c|c|}
\hline Parameter & Value \\
\hline Topology & Cluster \\
\hline Nodes & $\begin{array}{c}\text { 9 Node Sensor, } 3 \text { Cluster } \\
\text { Head, 1 PAN Coordinator }\end{array}$ \\
\hline BO & $3,4,5,6,7,8,9,10$ \\
\hline SO & $3,4,5,6,7,8,9,10$ \\
\hline Trafficload & $0.1,0.2,0.3,0.4,0.5$ \\
\hline Data Rate & $0.6,0.7,0.8,0.9,0.1$ \\
\hline Packet Size & $250 \mathrm{Kbps}$ \\
\hline
\end{tabular}

Table 2 below shows the BO and SO type of node and the value for each series, which contains the value of the throughput of the various configurations SO and BO are generated from each traffic load.

Table 2.BO and SO Conffiguration

\begin{tabular}{|c|c|c|c|c|c|}
\hline Series & BO=SO PAN & BO CH & SO CH & BO Node & SO Node \\
\hline 1 & 10 & 10 & 9 & 9 & 8 \\
\hline 2 & 9 & 9 & 8 & 8 & 7 \\
\hline 3 & 8 & 8 & 7 & 7 & 6 \\
\hline 4 & 7 & 7 & 6 & 6 & 5 \\
\hline 5 & 6 & 6 & 5 & 5 & 4 \\
\hline 6 & 5 & 5 & 4 & 4 & 3 \\
\hline 7 & 4 & 4 & 3 & 3 & 2 \\
\hline 8 & 3 & 3 & 2 & 2 & 1 \\
\hline
\end{tabular}


Figure 4 shows the maximum value of the overall throughput at various values trafficload.

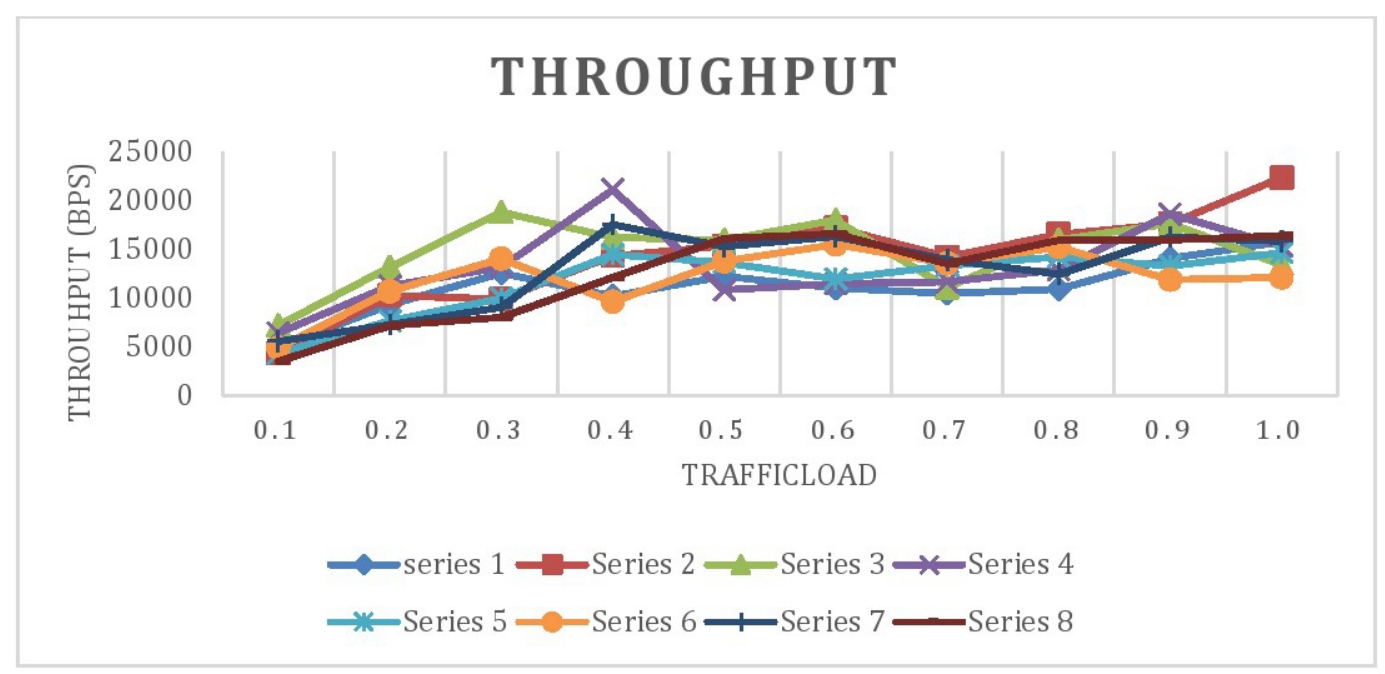

Figure 4. Adaptive Traffic Graph

To implement adaptive traffic then will be taken at each trafficload maximum throughput value. Table 3 shows the maximum throughput on each trafficload.

Table 3. SO,BO each Trafficload

\begin{tabular}{|c|c|}
\hline Trafficload & BO,SO \\
\hline 0,1 & 8 \\
\hline 0,2 & 8 \\
\hline 0,3 & 8 \\
\hline 0,4 & 7 \\
\hline 0,5 & 8 \\
\hline 0,6 & 8 \\
\hline 0,7 & 9 \\
\hline 0,8 & 9 \\
\hline 0,9 & 7 \\
\hline 1 & 9 \\
\hline
\end{tabular}

Throughput is an important parameter to determine the quality of performance of a network. The higher the quality the better the throughput performance of the network. Figure 5 shows the comparison of throughput between adaptive and non-adaptive traffic with $\mathrm{SO}=\mathrm{BO}=10$.From Figure 5 can be seen as a whole WSN with adaptive traffic has a higher throughput, this happens because the adaptive traffic can adapt to trafficload, so that in 
each value trafficload obtain maximum throughput. Non-adaptive does not have the ability to adapt, the value of SO and BO at every trafficload always the same so it does not get the value of the maximum throughput.

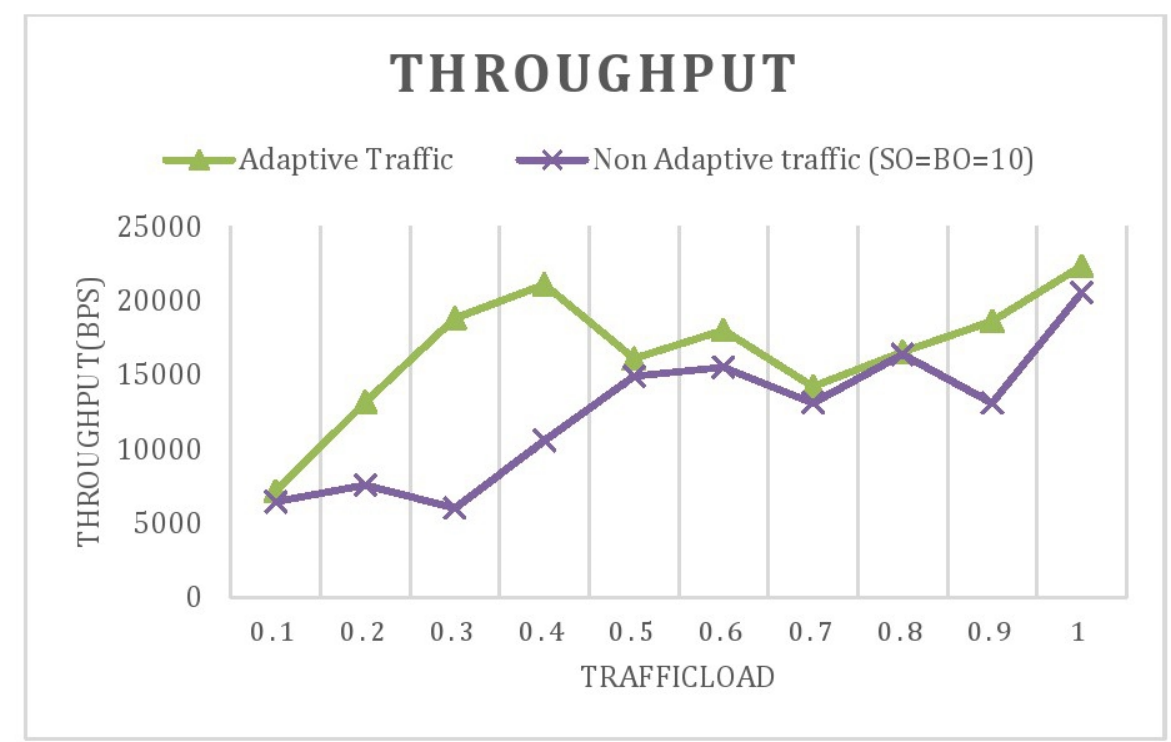

Figure 5.Traffic Load vs. Throughput

Energy consumption is influenced by the large SO and BO. Figure 6 shows the comparison of energy consumption between adaptive and nonadaptive traffic with $\mathrm{SO}=\mathrm{BO}=10$. The energy consumption in adaptive traffic is smaller than that of in the non-adaptive. Adaptive traffic implement the concept of coordinated sleeping so that there is a difference in the values of $\mathrm{BO}$ and SO PAN, Cluster Head and sensor nodes.

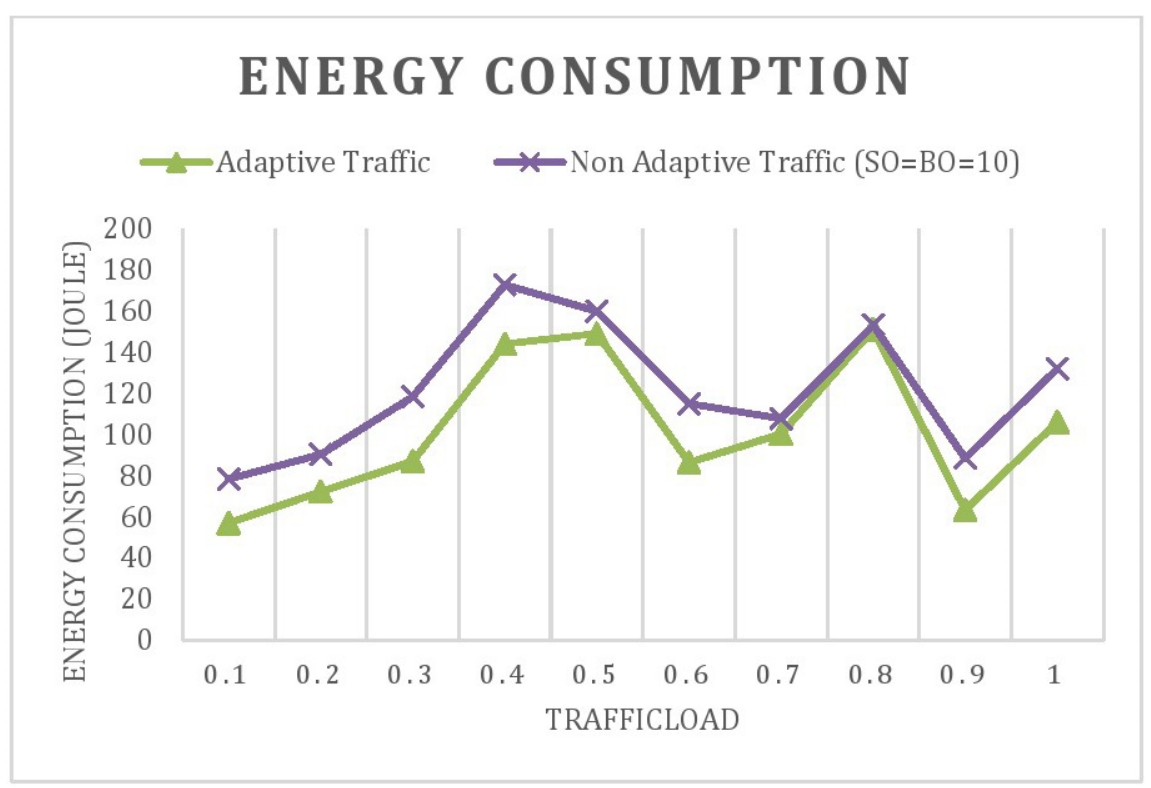

Figure 6. Traffic Load vs. Energy Consumption 
In addition, the $\mathrm{BO}$ and $\mathrm{SO}$ on the cluster head and sensor nodes are not equally so in the superframe are hasn't active parts. Since there are nonactive part of it can save energy consumption. In the non-adaptive value of SO and $\mathrm{BO}$ are the same so that the entire active superframe resulting in greater energy consumption. At 0.9 trafficload throughput generated large but little energy consumption, this happens because in addition to throughput is affected by the energy consumption of packet sent.

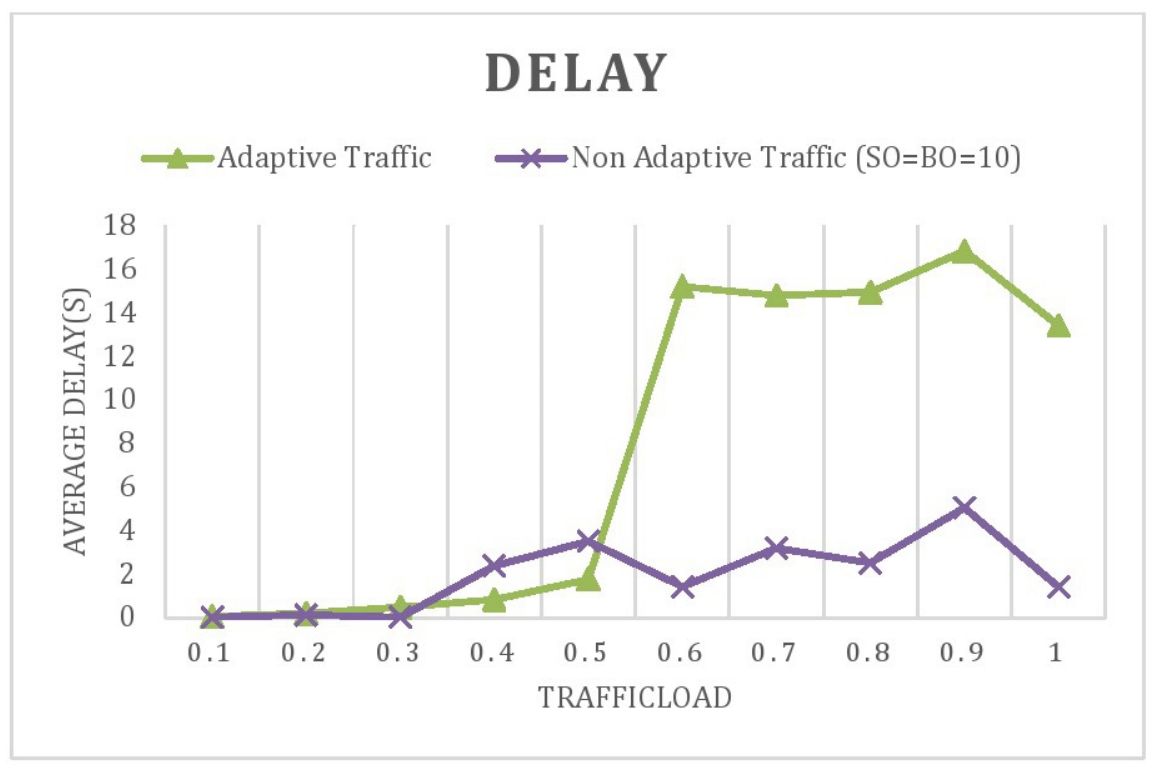

Figure 7.Traffic Load vs. Average Delay

Figure 7 shows the comparison of average delay between adaptive and non-adaptive traffic with $\mathrm{SO}=\mathrm{BO}=10$.The average delay on the non-adaptive trafficload 0.1 to 0.5 is almost the same, but the trafficload 0.5 to 1 on adaptive traffic delay value increased dramatically. Trafficload affect the value of the interval, the higher the value of trafficload, the lower interval value, so the more often a source node sends the data to the destination node. More frequent data transmission delay will also increase because the density of traffic. Moreover, in applying the concept of adaptive traffic coordinated sleeping, superframe have adequate non-active parts. In adaptive traffic and coordinated sleeping, the data transmission will be delayed if coincide with the non-active superframe part, thus it will wait till find the next active superframe. The more differences value of of BO and SO, the greater the resulting delay. However, there is no non-active partin the non-adaptive traffic,thus the node can send data without delay because another node is always in an active state so that resulting small delay value.

\section{CONCLUSION}

The combination of adaptivetraffic algorithms and coordinated sleeping is the solution that we propose to make energy savings in the WSN. We combine the concept of adaptive traffic and coordinatedsleeping concept 
to produce a good network performance with low energy consumption.To determine the quality of the combination of adaptive algorithms sleeping coordinated traffic and we compare it with the non-adaptive WSN. The experiment results show that the performance of traffic adaptive algorithms and coordinated sleeping is greater in throughput and more efficient in energy consumption, but has a weakness, namely the delay. Value of traffic delay on the combination of adaptive traffic algorithms and coordinated sleeping greater than non-adaptive. The higher the value of delay, network quality has declined. So that further research needs to be done to reduce the delay to get better network quality.

\section{REFERENCES}

[1] Kazuo MORI, Katsuhiro NAITO, and Hideo KOBAYASHI."Adaptive Backoff Control Method for Traffic Adaptive Active Period Control in Cluster-based IEEE 802.15.4 WSNs", Vehicular Technology Conference Fall (VTC 2009-Fall), 2009 IEEE 70th.

[2] Kazuo MORI, Katsuhiro NAITO, and Hideo KOBAYASHI."Traffic Adaptive Active Period Control with Adaptive Backoff Window for Clusterbased IEEE 802.15.4 Wireless Sensor Networks",International Conference onTelecommunications, 2009. ICT '09.

[3] Marwa S, Wail M,YaserE,Muneer Y ."Optimal Beacon and Superframe Orders in WSNs ", Jordan University of Science and Technogy, Jordan, 2013.

[4] Wei Ye, John Heidemann, and Deborah Estrin, "Medium Access Control With Coordinated Adaptive Sleeping for Wireless Sensor Networks", IEEE/ACM Transaction on Networking, VOL. 12, NO. 3, June 2004.

[5] Caishi Huang, Chin-Tau Lea, Albert Kai-Sun Wong, "On Mobility and Sleep-Mode Power-Saving Mechanisms in IEEE 802.16e/m Mobile Networks", IEEE Systems Journal, Issue: 99, January 2014.

[6] Irfan Al-Anbagi, MelikeErol-Kantarci, Hussein T. Mouftah, "A Traffic Adaptive Inter-Cluster Head Delay Control Scheme in WSNs", IEEE Symposium on Computers and Communications (ISCC), July 2013.

[7] ShuguoZhuo, Zhi Wang, Ye-Qiong Song, Zhibo Wang,Luis Almeida, "iQueue-MAC: A Traffic Adaptive duty-cycled MAC Protocol With Dynamic Slot Allocation", 10th Annual IEEE Communications Society Conference on Sensor, Mesh and Ad Hoc Communications and Networks (SECON), New Orleans LA, June 2013. 